\title{
Promoting neuroplasticity and neuropsychological functioning in frailty through an app-based sensorimotor training: study protocol for a randomized trial
}

Florian Beier ${ }^{1}$, Martin Löffler ${ }^{1}$, Frauke Nees ${ }^{1,2}$, Lucrezia Hausner ${ }^{1,3}$, Lutz Frölich ${ }^{3}$ and Herta Flor ${ }^{1,4^{*}}$ (D)

\begin{abstract}
Background: Frailty is characterized by an age-related decline in multiple physiological systems, leading to a high vulnerability to stressors, adverse health outcomes, and low quality of life. Neuroscientific models of pathological aging emphasize the loss of sensorimotor stimulation and reduced neuromodulatory capacities as core processes in age-related cognitive and bodily decline, which may be associated with maladaptive plastic changes in the brain. We plan to increase sensorimotor stimulation in frail persons through a newly developed app-based training program and link the training trials to biological and psychological correlates of age-associated vulnerability and health indices.

Methods: We will conduct a randomized trial, applying an app-based sensorimotor home training $(N=30)$ in people suffering from frailty. An app-based relaxation training will serve as an active control condition $(\mathrm{N}=30)$. Both interventions will last for 90 days each. The sensorimotor training includes unimodal and multimodal sensory discrimination tasks in the visual, auditory, and tactile domain, as well as sensorimotor precision tasks. The tasks will be implemented using an adaptive training algorithm and enriched with motivational components embedded in a virtual training environment. We expect a pre-post reduction of frailty status and associated functional decline related to refinement of representational maps within the sensorimotor system and improved sensorimotor function such as extremity function. Secondary analyses will study the influence of BDNF genotype as moderating variable. Additional outcomes will include measures of perceptual and cognitive functioning, quality of life as well as BDNF serum levels. Measurements will take place before training (baseline), after 60 days (assessment 1), and at the end of the training after 90 days (assessment 2).
\end{abstract}

\footnotetext{
*Correspondence: herta.flor@zi-mannheim.de

${ }^{1}$ Institute of Cognitive and Clinical Neuroscience, Central Institute of Mental

Health, Medical Faculty Mannheim, Heidelberg University, Square J5, 68159

Mannheim, Germany

${ }^{4}$ Department of Psychology, School of Social Sciences, University of

Mannheim, Mannheim, Germany

Full list of author information is available at the end of the article
}

(c) The Author(s). 2021 Open Access This article is licensed under a Creative Commons Attribution 4.0 International License, which permits use, sharing, adaptation, distribution and reproduction in any medium or format, as long as you give appropriate credit to the original author(s) and the source, provide a link to the Creative Commons licence, and indicate if changes were made. The images or other third party material in this article are included in the article's Creative Commons licence, unless indicated otherwise in a credit line to the material. If material is not included in the article's Creative Commons licence and your intended use is not permitted by statutory regulation or exceeds the permitted use, you will need to obtain permission directly from the copyright holder. To view a copy of this licence, visit http://creativecommons.org/licenses/by/4.0/ The Creative Commons Public Domain Dedication waiver (http://creativecommons.org/publicdomain/zero/1.0/) applies to the data made available in this article, unless otherwise stated in a credit line to the data. 
Discussion: In our randomized trial, we aim to characterize a multidimensional concept of frailty and to target maladaptive behaviors and neuroplasticity using an app-based sensorimotor training. This type of intervention might provide further knowledge and new possibilities for preventing decline and preserving function in older adults.

Trial registration: ClinicalTrials.gov NCT03666039. Registered 11 September 2018 - Retrospectively registered. Protocol version: Version 4 revised (issue date: 19 May 2021).

Keywords: Frailty, Neuroplasticity, Training app, Sensorimotor functioning, Aging

\section{Background}

The term "frailty" refers to a clinical condition which is characterized by an increased vulnerability to stressors and poor health outcomes resulting from a cumulative decline in multiple physiological systems, such as the musculoskeletal, endocrine, and cardiovascular system $[1,2]$. While a continuous decrease in physiological capacity also occurs with normal aging, this decrease is accelerated and exacerbated in frailty [3]. Neuroscientific models of pathological aging suggest that the brain might play a major role in determining healthy or pathological aging $[4,5]$. For instance, age-related gray matter reductions in the frontal $[6,7]$ and medial-temporal $[8$, 9] regions have been associated with reduced gait and memory performance, while white matter atrophy in the corpus callosum is suggested to affect bihemispheric communication [10, 11]. From a functional perspective, aging is related to reduced neural differentiation and selectivity of perceptual $[12,13]$ and motor [14] representations. These altered representations in turn are thought to be involved in an age-related decline in cognitive performance, such as memory $[15,16]$ and sensorimotor performance, including upper extremity function, gait and balance $[17,18]$. In this context, an important determinant is "disuse" of the brain, characterized by a reduction of perceptual inputs, motor actions, and cognitive stimulation that are required to refine existing skills and acquire new skills [5]. When people age they tend to stereotype and simplify behaviors and the brain is likely to adapt to these less complex behaviors by simplifying the underlying neuronal representations such as cortical sensorimotor maps $[4,5]$. On the neuronal level, "disuse" of the brain is thought to lead to negative changes in neuronal metabolism, such as neurotransmitter production and function [19] and neuronal architecture, including the elaboration of dendrites, spines, and synapses [20]. Therefore, the fidelity and reliability of cortical representations is thought to decline, resulting in noisy neuronal processing in sensory and motor systems, which in turn might promote maladaptive behaviors such as motor instability, coordination deficits, movement slowing, inactivity, and social isolation. Low activity levels lead to an increase in peripheral and bodily symptoms, such as a loss of muscle mass, which would increase the risk of fall and fracture [21]. Together, these interrelated factors create a self-reinforcing downward spiral of altered brain function, physical disability and age-related functional decline.

Therefore, we assume that the brain might be at the center of the vicious cycle of frailty, starting with an initial impairment and leading to an accelerated decline of physical function. As the brain is a highly plastic organ that shows adaptive as well as maladaptive plasticity [22], it might be an optimal target for innovative interventions that utilize principles of neuroplasticity to delay or even reverse cortical and behavioral age-related changes.

To date, the majority of interventional studies in older frail individuals examine physical exercise protocols including aerobic [23] and muscular strengthening exercises [24]. Physical exercise has been suggested to improve physical performance such as muscle strength, balance, and gait speed [25], but also to enhance brain health and plasticity [26]. Physical therapy in pre-frail individuals was found to increase reduced plasma levels of brain-derived neurotrophic factor (BDNF), a neuronal growth factor involved in neurogenesis and synaptogenesis [27], which suggests a key role of neuromodulatory factors in mediating the syndrome of frailty [28]. However, only few of these studies have directly evaluated the influence of physical exercise on frailty itself [29] and the optimal procedure of how to prevent or reverse the syndrome of frailty is still a matter of debate [30, 31]. Moreover, despite the evidence suggesting a close link between structural as well as functional brain changes and physical decline during aging $[17,18]$, interventional studies targeting the relationship between brain structure and function and frailty are surprisingly rare. In healthy adults, neuroplasticity-oriented programs, including intensive sensory, cognitive, or motor stimulation, were shown to have the potential to strengthen neuromodulatory systems, promote beneficial neuroplasticity in cortical representations, and improve neurocognitive skills that decline with aging [32-34]. As motivational and affective processes seem to be less 
affected by maladaptive plasticity [35], these processes provide useful means to enhance training compliance and success. With respect to the multi-system decline observed in frail individuals, computerized neuroplasticityoriented applications using virtual environments can be more useful because they can stimulate several systems at the same time, can give immediate feedback and permit the use of everyday activities including bodily activation that are relevant for the participant's life [36]. Therefore, we describe a randomized trial in which we use an appbased multimodal sensorimotor training in frailty.

\section{Objectives of the study}

Our hypothesis is that intensive forms of plasticityoriented training can improve frailty. Our goal is to enhance relevant input to the sensory and motor brain systems in order to reverse structural and functional correlates of maladaptive neuroplasticity in cortical representational maps. We aim at counteracting the "disuse" of the brain, which should lead to positive changes in neuronal metabolism and architecture and thus to an increased fidelity and reliability of cortical representations and less noisy neuronal processing. In turn, we expect these neuroplastic changes to promote cognitive, physical, and sensorimotor function, leading to an improvement in frailty status and frailty-related health indices.

Based on previous plasticity-oriented training studies in healthy subjects [32-34], our training approach will consist of a multimodal training protocol including unimodal sensory discrimination and bimodal sensory integration tasks in the visual, auditory, and tactile domain as well as a sensorimotor precision tasks (cf. [37]). The training tasks will be implemented in an app-based manner and will be embedded in a motivating virtual environment including personally relevant reinforcers to counteract the age-related reduction in motivational drive and increase adherence to and efficacy of the training. To evaluate the specificity of the sensorimotor approach, we will implement a randomized controlled trial with an app-based relaxation training serving as an active control group.

We hypothesize that the sensorimotor training will have superior effects on frailty status compared with the control training, as reflected by a pre-to-post reduction in frailty indices and an improvement in frailty-related everyday functioning. On the neuronal level, we expect a refinement of sensorimotor representation maps and an increase in neuronal efficiency in the brain. Furthermore, we expect that such neuroplastic changes will be mirrored by a pre-to-post increase in BDNF serum levels. In addition, we will compare frailty-associated outcomes between BDNF genotypes that were shown to differ in terms of both BDNF secretion and the magnitude of training-related improvement $[27,38]$. In the sensorimotor domain, we expect an increase in movement speed as well as an improvement in lower extremity motor function such as balance, and upper extremity motor function such as dexterity. With regard to sensory abilities, we expect an increase in visual, tactile, and auditory performance scores. Lastly, we expect the sensorimotor training approach to enhance psychological wellbeing, as reflected by an increased perceived health status and quality of life.

\section{Methods \\ Design}

This randomized controlled trial will compare a tabletbased sensorimotor training (experimental group) and a tablet-based relaxation training (control group) in subjects suffering from frailty. Both conditions are conceptualized as a 90-day home-based intervention. Assessments will take place (1) before (baseline), (2) after 60 days (assessment 1 ) and (3) at the end of training (90 days; assessment 2) at the Institute of Cognitive and Clinical Neuroscience, Central Institute of Mental Health, Mannheim.

\section{Study population and recruitment}

The expected recruitment of participants is shown in Fig. 1. Participants will be recruited from the general population as well as from geriatric hospitals via personal contact, newspaper advertisements, leaflets and online announcements. Prior to the invitation to the $\mathrm{CIMH}$, potential subjects will be pre-screened for general eligibility via telephone interviews. Specific eligibility criteria will be tested and general eligibility criteria will be confirmed during the first examination appointment.

The inclusion criteria will be an age of 65 to 95 years and the presence of at least one of the five frailty phenotype criteria [39], i.e. self-reported unintentional weight loss of $>5 \mathrm{~kg}$ in the prior year, self-reported exhaustion, low level of physical activity specified in $\mathrm{kcal} / \mathrm{week}$, muscle weakness as measured by low grip strength using a Jamar hand dynamometer, and slowness by slowed gait speed over $4.57 \mathrm{~m}$. According to the phenotype model, the presence of one or two criteria is considered as prefrailty while the presence of three or more criteria is considered as frailty [39]. We will include pre-frail as well as frail persons to investigate neuroplasticity mechanisms across a broader range of age-related functional decline, as the pre-frail stage is considered to describe a condition at high risk of progression to frailty $[40,41]$ and may be more amenable to change. Exclusion criteria will be: acute bone fractures within the last 3 months, immobility, paralysis or confinement to bed; stroke or neurological disorders with major cognitive or physical impairments; dementia; myocardial infarction within the last 6 months; life-time prevalence of mental disorders 


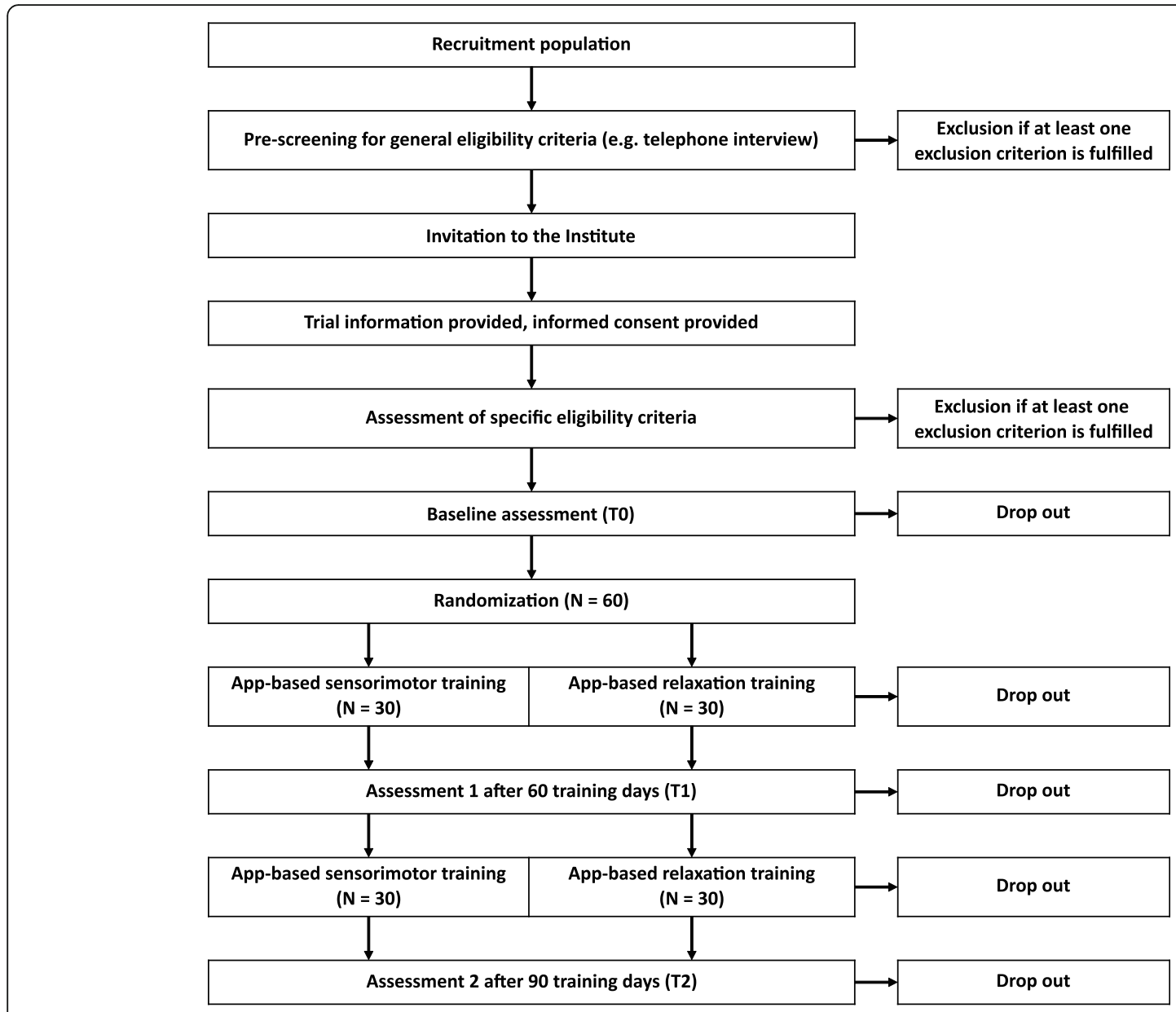

Fig. 1 Expected flow of participants and study design

such as schizophrenia and other psychotic disorders, bipolar disorder, obsessive-compulsive disorder, posttraumatic stress disorder, drug or alcohol addiction; current severe major depression or other acute axis 1 mental disorders; current intake of benzodiazepines or antipsychotics; vitamin B12-, folate- or thyroidstimulating hormone deficiency. Specific exclusion criteria will be examined during the first visit and include: cognitive impairment defined as a Mini Mental State Examination (MMSE) score of $\leq 24$; severe impairments in sensory abilities (i.e. visual acuity of $<0.1$; mechanical detection threshold of $>512 \mathrm{mN}$; severe or profound hearing loss according to the WHO, defined as a mean hearing threshold of $>60 \mathrm{~dB}$; severe tinnitus symptomatology).

\section{Procedure}

Subjects fulfilling the general eligibility criteria as determined through the pre-screening will be invited for baseline assessments. During the first visit at the CIMH, subjects will provide written consent for participation. Specific eligibility criteria will be examined by a trained psychologist and a trained psychiatrist specialized in geriatric psychiatry. Individuals fulfilling the diagnostic criteria will then undergo behavioral, neuropsychological, and neurophysiological assessments conducted by the psychologist, and a physical and neuropsychological examination as well as the assessment of the medical history and medication conducted by the psychiatrist. Blood samples will be collected and conserved for analysis of BDNF serum levels and genotypes. After baseline assessments, subjects will be introduced to the respective training procedure by a psychologist. Support during the home-based treatment is also provided. After 60 and 90 days, the assessment of sensorimotor, neurocognitive and neurophysiological functioning will be repeated at the CIMH. Neuropsychological and physical examination including collection of blood samples will be repeated after 60 days. Prior to and after the training, participants will be asked for treatment expectation and evaluation, respectively, using five questions adapted from [42] to uncover motivational and affective aspects of the training. Subjects will not be paid for participation, but will be compensated for their travel costs. 


\section{Randomization and blinding}

Subjects will be randomly allocated on a 1:1 ratio to either the experimental or control condition. The randomization schedule will be generated electronically by a researcher not involved in the assessments. Group allocations will be kept in sequentially numbered sealed opaque envelopes. Participants will be informed about the random allocation procedure. Blinding of the investigator will not be possible because the investigator will conduct parts of the assessments and training procedures.

\section{Sample size}

Previous studies in healthy older humans using sensory training have achieved promising results with medium effect sizes and sample sizes of 41 to 53 subjects [5, 32]. Given that our training includes a multimodal stimulation approach and focuses on motivational enhancement within a virtual training environment, we assume that in our participants the effect of the experimental training program would exceed a medium effect size. To estimate sample size, we use a repeated measures design with one between-factor with two groups and one within-factor with three assessment points [43]. Assuming a medium effect size of Cohen's $f=0.25$, 95\% statistical power, a correlation of 0.50 between the dependent measurements, and a two-sided alpha error level of .05, we therefore would need 22 participants in each group. To account for a drop-out rate of about $25 \%$ during the training, we will include 30 subjects per group (60 in total).

\section{Interventions}

\section{Experimental condition}

The interactive tablet-based sensorimotor intervention will consist of three consecutive training phases with each phase comprising 30 days (90 days in total). The first phase will consist of unimodal sensory discrimination tasks in the visual, auditory and tactile domain to increase distinctiveness and acuity of cortical sensory representations. In the second phase, subjects will be trained on bimodal sensory integration using visualauditory, visual-tactile and auditory-tactile tasks. Bimodal sensory integration will require subjects to process stimuli of two different perceptual modalities and judge whether the two stimuli are synchronous or not. These tasks are assumed to increase temporal acuity for multisensory integration and to promote plasticity in perceptual integration brain networks [44, 45]. Unimodal and bimodal stimuli are presented through the tablet involving explicit answers on the tablet's touch screen. The third phase will require participants to process bimodal sensory information in order to control a sensorimotor response, in the form of visual-auditory guided cycling, visual-tactile determined grasping, and auditoryvisual controlled hands coordination. In these sensorimotor integration tasks, sensory input must be interpreted and integrated in terms of the current state of the motor system to continuously adjust motor commands and behavior [46]. These tasks aim at improving motor acuity, i.e. to increase precision and reduce variability of motor performance by increasing the signal-to-noise ratio in sensorimotor brain networks [47, 48]. For tactile, cycling, and grasping tasks, we will use external devices that are wirelessly connected to and controlled by the tablet (i.e. a Braille display device for tactile tasks, a customized ergometer for cycling tasks, and a handgrip dynamometer for grasping tasks).

Each daily training session will last $30 \mathrm{~min}$ allowing self-determined breaks in between. The training tasks will be embedded in a container application featuring a virtual environment of a gaming nature [34]. Performing the daily training tasks, participants can earn tickets to progress on a virtual journey throughout European cities. To enhance training motivation and efficacy, the program will include a customized application environment as well as personally relevant reinforcers (pictures, sounds) individually embedded in the tasks. To enhance training motivation, task difficulty will be dynamically adapted to provide a positive feedback percentage of 70$80 \%$. Accordingly, the adaptive difficulty manipulation should produce sustained sensory and sensorimotor challenges, thereby promoting lasting neural changes and transfer effects [34, 49]. For monitoring and analysis purposes, training data including duration and achievements will be automatically collected by the app and sent to a dedicated server on a daily basis.

\section{Control condition}

The control intervention will be a self-developed tabletbased relaxation training encompassing daily 30-min sessions across a total training duration of 90 days, which do not include any of the critical features of the sensorimotor training app (i.e. multimodal sensorimotor training, adaptive algorithm, personalized feedback) using stimulating and variable exercises. Training sessions will require participants either to watch relaxation videos consisting of nature scenes or to follow verbally guided relaxation exercises, alternating on a regular basis. Relaxation exercises encompass various relaxation techniques, such as autogenic training, breathing meditation, mindfulness practice, or imaginary journeys. At the end of each session, participants complete a short questionnaire about their affective state including items from the German version of the Positive and Negative Affect Schedule (PANAS) [50, 51]. Training data collected by the app include responses to the post-training 
questionnaire as well as total amount of time engaged in the daily training.

\section{Outcome measures and biological moderators}

The assessments will be performed according to the structure depicted in Table 1.

\section{Frailty status}

Comparison with other training studies will be possible by using the frailty phenotype [39] as a measure of frailty status as primary outcome. Additionally, we will assess the frailty index $[52,53]$, which is calculated as the ratio of the number of deficits present out of a total number of 40 deficits assessed, including previous diseases, disability, psychosocial risk factors as well as physical and cognitive impairments [53]. Thus, the frailty index consists of a continuous score between 0 and 1 with higher values representing more pronounced frailty and increased susceptibility to adverse health outcomes [53]. While some studies demonstrated that frailty scores obtained from the two measures are comparable [54, 55], others have suggested that the frailty index might discriminate better at the lower to middle end of the frailty continuum [56] and might be more sensitive to measure change after an intervention [57]. Therefore, we will use both measures in a complementary rather than comparative manner [58].

\section{Brain plasticity in the sensorimotor and somatosensory system}

In order to track correlates of functional and maladaptive neural plasticity, we will measure neural processing and cortical representations in the sensorimotor as well as somatosensory system using functional magnetic resonance imaging (MRI).

Sensorimotor brain activation will be investigated using a motor sequence task encompassing three different motor exercises of varying complexity [59]. During each trial, participants will have to perform a sequence of button presses with their right hand using an MRIcompatible keyboard with five keys that are numbered from 1 to 5 and correspond to the thumb, index finger, middle finger, ring finger, and little finger, respectively. The task consists of three different exercises requiring repetitive tapping of a certain sequence: the "FINGER" condition consists of repetitive tapping of key no. 2 with

Table 1 Overview of outcomes, outcome measures, instruments and assessment time points

\begin{tabular}{|c|c|c|c|}
\hline Outcomes & Outcome measures & Instruments & $\begin{array}{l}\text { Assessment } \\
\text { time points }\end{array}$ \\
\hline Personal information & - Age, sex, education, clinical history, medication & - Self reports & $\cdot \mathrm{T} 0, \mathrm{~T} 1, \mathrm{~T} 2$ \\
\hline Frailty assessments & $\begin{array}{l}\text { - Frailty status (pre-frail, frail) } \\
\text { - Frailty Index }\end{array}$ & $\begin{array}{l}\text { - Frailty Phenotype } \\
\text { - Frailty Index }\end{array}$ & $\begin{array}{l}\cdot \mathrm{T} 0, \mathrm{~T} 1, \mathrm{~T} 2 \\
\cdot \mathrm{T} 0, \mathrm{~T} 1, \mathrm{~T} 2\end{array}$ \\
\hline $\begin{array}{l}\text { Brain plasticity in the sensorimotor } \\
\text { and somatosensory system }\end{array}$ & $\begin{array}{l}\text { - Structural and functional parameters of cortical } \\
\text { sensorimotor and somatosensory maps } \\
\text { - MEP peak-to-peak amplitude and latency }\end{array}$ & $\begin{array}{l}\text { - fMRI motor sequence task, fMRI } \\
\text { somato-sensory mapping task } \\
\text { - TMS at left primary motor cortex }\end{array}$ & $\begin{array}{l}\cdot \mathrm{T} 0, \mathrm{~T} 1 \\
\cdot \mathrm{T} 0, \mathrm{~T} 1\end{array}$ \\
\hline Biological markers of neuroplasticity & - BDNF serum blood levels (ng/ml) & - Collection of blood samples & $\cdot \mathrm{T0}, \mathrm{T} 1$ \\
\hline Sensorimotor performance & $\begin{array}{l}\text { - SPPB (total score) } \\
\text { - CTSIB (total score) } \\
\text { - PPT (means) }\end{array}$ & $\begin{array}{l}\text { - SPPB } \\
\text { - CTSIB } \\
\text { - PPT }\end{array}$ & $\begin{array}{l}\cdot \mathrm{T} 0, \mathrm{~T} 1, \mathrm{~T} 2 \\
\cdot \mathrm{T} 0, \mathrm{~T} 1 \\
\cdot \mathrm{T} 0, \mathrm{~T} 1, \mathrm{~T} 2\end{array}$ \\
\hline Sensory functioning & $\begin{array}{l}\text { - Visual acuity } \\
\text { - Visual contrast sensitivity } \\
\text { - Tactile grating thresholds } \\
\text { - Fine-touch thresholds } \\
\text { - Hearing thresholds }\end{array}$ & $\begin{array}{l}\text { - FrACT } \\
\text { - FrACT } \\
\text { - JVP domes } \\
\text { - Von-Frey filaments } \\
\text { - Audiometer }\end{array}$ & $\begin{array}{l}\cdot \mathrm{T} 0, \mathrm{~T} 1, \mathrm{~T} 2 \\
\cdot \mathrm{T} 0, \mathrm{~T} 1, \mathrm{~T} 2 \\
\cdot \mathrm{T} 0, \mathrm{~T} 1, \mathrm{~T} 2 \\
\cdot \mathrm{T} 0, \mathrm{~T} 1 \\
\cdot \mathrm{T} 0, \mathrm{~T} 1\end{array}$ \\
\hline Cognitive testing & $\begin{array}{l}\text { - MMSE (total score) } \\
\text { - Mental and motor response speed } \\
\text { - Top-down attentional control } \\
\text { - Visuospatial working memory capacity } \\
\text { - Executive functioning }\end{array}$ & $\begin{array}{l}\cdot \text { MMSE } \\
\cdot \text { RTI } \\
\cdot \text { AST } \\
\cdot \text { SSP } \\
\cdot \text { IED }\end{array}$ & $\begin{array}{l}\cdot \mathrm{T} 0, \mathrm{~T} 1, \mathrm{~T} 2 \\
\cdot \mathrm{T} 0, \mathrm{~T} 1 \\
\cdot \mathrm{T} 0, \mathrm{~T} 1, \mathrm{~T} 2 \\
\cdot \mathrm{T} 0, \mathrm{~T} 1 \\
\cdot \mathrm{T} 0, \mathrm{~T} 1\end{array}$ \\
\hline Functional level and quality of life & $\begin{array}{l}\text { - Functional level in frailty } \\
\text { - Depression } \\
\text { - Overall health status and quality of life } \\
\text { - Functional capacity in everyday activities } \\
\text { - Falls self-efficacy } \\
\text { - Nutritional status }\end{array}$ & $\begin{array}{l}\text { - } \text { FEFA } \\
\text { - CES-D } \\
\text { - SF-36, EQ-5D-5L } \\
\text { - } \text { MKS } \\
\text { - FES-I } \\
\text { - } \text { MNA }\end{array}$ & $\begin{array}{l}\cdot \mathrm{T} 0, \mathrm{~T} 1, \mathrm{~T} 2 \\
\cdot \mathrm{T} 0, \mathrm{~T} 1 \\
\cdot \mathrm{T} 0, \mathrm{~T} 1, \mathrm{~T} 2 \\
\cdot \mathrm{T} 0, \mathrm{~T} 1 \\
\cdot \mathrm{T} 0, \mathrm{~T} 1, \mathrm{~T} 2 \\
\cdot \mathrm{T} 0, \mathrm{~T} 1\end{array}$ \\
\hline
\end{tabular}

AST Attention Switching Task, BDNF Brain-derived neurotrophic factor, CES-D Center for Epidemiologic Studies Depression Scale, CTSIB Clinical Test of Sensory Integration of Balance, EQ-5D-5L EuroQol-5D-5L, FEFA Frail Elderly Functional Assessment, FES-I Falls Efficacy Scale - International Version, fMRI Functional magnetic resonance imaging, FrACT Freiburg Vision Test, IED Intra-Extra Dimensional Set Shift, MEP Motor-evoked potential, MKS Marburg Competency Scale (Marburger Kompetenz Skala), MMSE Mini Mental State Examination, MNA Mini Nutritional Assessment, PPT Purdue Pegboard Test, RTI Reaction Time, SF-36 Short Form-36 Health Survey, SPPB Short Physical Performance Battery, SSP Spatial Span, T0 Baseline assessment, T1 Assessment 1 after 60 days, T2 Assessment 2 after 90 days, TMS Transcranial magnetic stimulation 
the index finger, the "SIMPLE" condition consists of the sequence 1-2-3-4-5, and the "COMPLEX" condition consists of the sequence 1-3-5-2-4. The task will be carried out in a block-wise manner consisting of alternating movement and rest blocks.

Functional MR scans will be recorded using a $3 \mathrm{~T}$ Magnetom Trio system (Siemens, Erlangen, Germany) and a 32-channel head coil. Using an EPI gradient echo sequence, we will collect 40 slices with $2.3 \mathrm{~mm}$ slice thickness, $\mathrm{TE}=22 \mathrm{~ms}, \mathrm{TR}=2100 \mathrm{~ms}, \quad \mathrm{FoV}=220 \times 220$ $\mathrm{mm}^{2}$, voxel resolution $=2.3 \times 2.3 \times 2.3 \mathrm{~mm}^{3}$.

To investigate neuronal reorganization in the somatosensory system, we will perform a spatial mapping of the finger representations in the primary somatosensory cortex (S1) using two different protocols of somatosensory stimulation. Stimuli will consist of pneumatic sensations of touch automatically applied at a frequency of $1 \mathrm{~Hz}$ through a custom-made pneumatic device [60]. In a first run, tactile stimuli to the five finger pads will be separately applied in a random block design interspersed with rest blocks. In a second run, we will apply a well validated phase-encoding paradigm in which fingers will be continuously stimulated with no rest blocks. This approach has been demonstrated to reveal highly reproducible maps of individual digits in S1 [61]. Stimulation will cycle through blocks of fingers in an ascending and descending order. For both somatosensory paradigms, we will use an EPI gradient echo sequence and collect 22 slices with $1.8 \mathrm{~mm}$ slice thickness, $\mathrm{TE}=22 \mathrm{~ms}$, $\mathrm{TR}=$ $1500 \mathrm{~ms}$, FoV $=220 \times 220 \mathrm{~mm}^{2}$, voxel resolution $=1.2 \times$ $1.2 \times 1.8 \mathrm{~mm}^{3}$. In addition, we will acquire T1-weighted structural scans $(\mathrm{TE}=2.72 \mathrm{~ms}, \mathrm{TR}=1900 \mathrm{~ms}, \quad \mathrm{FoV}=$ $250 \times 250 \mathrm{~mm}^{2}$, voxel resolution $=0.8 \times 0.8 \times 0.8 \mathrm{~mm}^{3}$ ) to perform voxel-based morphometry.

\section{Neuronal efficiency in the sensorimotor brain system}

Age-related decline in sensorimotor performance has been linked to decreased neuronal efficiency in the sensorimotor system with respect to reduced motor cortex excitability [62, 63]. To investigate motor cortex excitability at baseline and after the intervention, we will perform single-pulse transcranial magnetic stimulation (TMS) at the left primary motor cortex (M1) and simultaneous recording of motor-evoked potentials (MEP) at the right abductor pollicis brevis. As measures of motor cortex excitability, we will calculate MEP peak-to-peak amplitudes and latencies. Prior to the stimulation, we will determine individual resting motor threshold (RMT), defined as the stimulation intensity at which at least 6 out of 10 MEPs reached a peak-to-peak amplitude of $\geq 50 \mu \mathrm{V}$ [64]. We will then conduct three stimulation runs at 100, 110 and $120 \%$ of the RMT intensity in pseudorandomized order. Each run will consist of 20 consecutive stimulations with an inter-stimulus interval of approximately $10 \mathrm{~s}$.

\section{Sensorimotor performance}

To measure lower extremity function we will use the Short Physical Performance Battery (SPPB) [65] comprising measures of balance, walking speed, and sit-tostand ability. Individual subtest scores range from 0 to 4 , resulting in a summary score ranging from 0 to 12 . Lower SPPB scores represent reduced physical abilities and have been associated with greater frailty [66] though previous studies demonstrated that physical activity interventions can result in improved SPPB scores [67].

We will use a modified version of the Clinical Test of Sensory Integration of Balance (CTSIB) [68] to estimate how well subjects can utilize vision, somatosensation, and vestibular information for the maintenance of postural stability. Subjects are required to maintain their feet side-by-side for $30 \mathrm{~s}$ with eyes open on a firm surface, eyes closed on a firm surface, eyes open on an unstable surface, and eyes closed on an unstable surface.

Upper extremity function will be assessed using the Purdue Pegboard Test (PPT) [69] requiring participants to place cylindrical metal pegs into holes either with the dominant, non-dominant or both hands simultaneously within a given time. In a fourth condition, participants have to combine pegs, washers and small tubes into a pre-defined assembly. Thus, the test measures fine and gross motor dexterity as well as coordination of hands, fingers, and arms [70] and poor PPT performance has been shown to be associated with age-related frailty [71].

\section{Sensory functioning}

In the sensory domain, we will assess visual, tactile, and auditory functioning. Visual testing will be carried out using the automated Freiburg Vision Test (FrACT) [72, 73]. For visual acuity testing, we will use Landolt Cs and Snellen Es of different size and orientation depicted on a computer screen in a pre-defined distance and under standardized lighting conditions. Visual contrast sensitivity will be assessed using Landolt Cs and gratings of different orientation and level of contrast.

Tactile discrimination performance will be tested in the form of a grating orientation task using hemispherical plastic domes (JVP Domes, Stoelting Europe, Dublin, Ireland), which have parallel bars and grooves of equal width on their surface (15 domes with a width range of 0.35 to $12 \mathrm{~mm}$ ). To determine grating orientation thresholds, gratings are manually applied by the experimenter to the subjects' skin (right index finger pad, right ring finger pad, right lower lip, in a randomized order) and subjects are to indicate the orientation of the grating (vertical, horizontal). 
Fine-touch thresholds will be evaluated by probing the fingertips of the dominant second and fourth digit as well as the back of the dominant hand using von Frey filaments (Marstocknervtest, Marburg, Germany). Touch forces range from $0.25 \mathrm{mN}$ to $512 \mathrm{mN}$ on a logarithmic scale. Thresholds will be determined by using a staircase procedure [74].

To assess auditory acuity, hearing thresholds within the frequency range from 0.125 to $8 \mathrm{kHz}$ will be determined in the form of an audiogram using a screening audiometer (MA 25, MAICO Diagnostics GmbH, Berlin, Germany). Based on a staircase procedure, single tones will be presented via headphones separately to the right and left ear in a counterbalanced sequence.

\section{Cognitive testing}

Previous research demonstrated that physical frailty might be associated with changes and impairments in a number of cognitive domains, such as episodic, semantic, and working memory [75], perceptual and psychomotor speed [75, 76], executive function [77] and topdown attention [78]. To assess cognitive abilities we will select four subtests of the Cambridge Neuropsychological Test Automated Battery (CANTAB; Cambridge Cognition (2019), www.cantab.com).

The Reaction Time (RTI) test assesses information processing speed, allowing for separate estimates of mental and motor response speed.

Using the Attention Switching Task (AST) we will examine top-down attentional control defined as the ability to flexibly switch attentional resources towards relevant information and inhibit irrelevant information. Dependent measures include response time and accuracy, switching cost and congruency cost.

Using the Spatial Span (SSP) test, we will assess working memory span to obtain an estimate of visuospatial working memory capacity.

Finally, we will investigate the integrity of frontostriatal pathways using the Intra-Extra Dimensional Set Shift (IED), which is a computerized version of the Wisconsin Card Sorting test. The IED is a test of executive functioning including rule acquisition and reversal as well as attentional set formation maintenance, shifting and flexibility of attention.

\section{Functional level and quality of life}

To assess function in frail elderly at a very low activity level, we will apply the Frail Elderly Functional Assessment (FEFA) [79], which has been demonstrated to be valid, reliable, and sensitive to change [80]. Using the German version of the Center for Epidemiologic Studies Depression Scale (CES-D), we will assess depressive symptoms experienced in the past week based on a 20item self-report scale [81, 82]. Overall health status and quality of life will be evaluated using the Short Form-36 Health Survey (SF-36) [83] and the EuroQol-5D-5L (EQ$5 \mathrm{D}-5 \mathrm{~L})$ [84]. The SF-36 is a widely used survey of quality of life in health economics and allows for evaluation of patient health on physical and mental health scales. The EQ-5D-5L quantifies health status in five dimensions and on a visual analogue scale ranging from 0 to 100 . To estimate functional capacity of the participants, we will use the self-report version of the Marburg Competency Scale (Marburger Kompetenz Skala, MKS) [85], which involves 30 questions on competence in typical everyday activities. Falls self-efficacy will be measured using the Falls Efficacy Scale - International Version (FES-I) [86], a self-report questionnaire assessing the level of concern regarding the possibility of falling when performing certain activities of daily living. To screen for malnutrition, we will use the Mini Nutritional Assessment (MNA) [87].

\section{BDNF serum level assessment and genotyping}

Blood samples collected by the study psychiatrist will be processed according to standard protocols and stored at $-80{ }^{\circ} \mathrm{C}$ until analysis. BDNF serum levels as a marker of synaptic plasticity will be measured using a highly sensitive fluorometric enzyme-linked immunoabsorbent assay (ELISA). For genetic analyses, we will extract DNA from blood samples. GWAS analysis will be performed on an Illumina GSA1.0 SharedCustom Content bead array according to the manufacturer's instructions. GenomeStudio 2.0 software will be used to determine BDNF genotypes and results will be exported in PLINK format.

\section{Data analysis and handling}

We will use mixed repeated measures analyses of variance for each of the outcome variables in order to assess the effect of the training at baseline, after 60 days, and after 90 days, as well as to unveil differences between the two groups. Significant time-by-group interactions will be further examined using post-hoc tests. To evaluate changes through the interventions, pre-post effect sizes will be computed and compared across interventions. The problem of missing data will be handled using multiple imputation.

Data collected in this project will be recorded, stored and analyzed anonymously. A list assigning codes to names will be stored separately under high security standards. Analysis of the data will be of scientific purpose only.

\section{Ethics and dissemination}

The study has been approved by the local Ethics Committee (Medical Ethics Committee II, Medical Faculty Mannheim, Heidelberg University; 2015-544 N-MA) according to the Declaration of Helsinki and has been 
registered under the trial number NCT03666039. If any important changes apply to the trial protocol, the trial registration will be updated. Any personal information of study participants will be stored and protected in accordance with the most recent General Data Protection Regulation of the European Union and will be monitored by the department's data protection commissioner. Direct access to data will be restricted to authorized representatives from the host institution and the regulatory authorities. The results of the study will be submitted for publication in a selected peer-reviewed journal on geriatrics and presented at relevant international conferences.

Participants will be informed about all procedures and written informed consent will be obtained. Any severe adverse events caused by our assessments or treatment protocols will be recorded and analyzed. During assessments, participants will be monitored by the investigator at all times and on a regular basis during the training procedures to maintain the opportunity to intervene if any unintended effects occur. A physician is available in cases of emergency. During measurements, the institute's liability insurance protects the participants against mishandling of the investigator.

\section{Discussion}

With the number of older people strongly increasing in almost all countries, frailty prevalence is expected to rise dramatically [2], placing a heavy burden on health and aged care systems $[88,89]$. As clinical studies in frail older patients are challenging and still rare, or yielded inconsistent results, procedures to reliably identify early onset stages of frailty as well as appropriate interventions to prevent disability and preserve physical functions are desperately needed.

The objective of our intervention is to increase relevant input into the sensorimotor system in order to engage competitive processes in the brain that refine the representations of sensory inputs and motor actions, thereby increasing the strength of cortical resources and reversing the maladaptive "disuse" of the brain [5]. Therefore, we expect our approach to promote sensorimotor and physical abilities, including motor stability, gait, and extremity function, and to reduce maladaptive behaviors, such as inactivity and social isolation, thereby leading to an improvement in frailty status.

Previous interventional studies in frailty included various types of intervention, such as physical exercise [24, 90, 91], nutritional intervention [92, 93], cognitive training [94], or geriatric assessment intervention [95, 96]. In particular physical exercise interventions demonstrated to be effective in reducing frailty and improving physical performance, while other interventions revealed only small or no training effects $[25,29]$. This heterogeneity of study results might be due to differences in the study protocols with respect to training protocol, intensity, frequency, delivering method, and outcome measures. Thus, the reasons why some interventions are effective while others are not still remain unclear. This impedes interpretability and generalizability of the findings [29].

Despite the fact that frailty is associated with a decline in multiple physiological systems [1], the role of the central nervous system and in particular the brain in the pathogenesis of frailty has been addressed in only a small number of studies and still remains unclear. In fact, evidence suggested a link between frailty and brain physiology. For instance, the physical frailty phenotype has been related to reduced cerebellar gray matter volume [97], and some of the phenotype criteria, particularly walking speed, have been associated to regional brain volumes in the prefrontal cortex [6] and corpus callosum [98]. Moreover, evidence suggested a clear link between frailty and cognition, such that the presence of frailty can predict cognitive impairment and neurodegenerative diseases, such as dementia, within a few years $[99,100]$. In this context, neurotrophic factors, including BDNF, may play an important role in survival, due to their role in promoting the differentiation of new neurons and synapses [101] and in preventing neuronal death during stress [102]. In fact, physical therapy intervention was found to increase plasma BDNF levels in pre-frail elderly women, suggesting that BDNF may be a key mediator in the pathophysiology of frailty [28]. However, interventional studies in frailty focusing on training effects in the brain are still lacking.

With the current trial, we will employ a multidimensional characterization of frailty, including neurophysiological, physical, sensorimotor, cognitive, and functional variables, which will enable us to investigate training effects on frailty status as well as on frailty-related outcomes. Through our intervention, we will target core central nervous processing in the sensorimotor system, by which we intend to create a link between structural, functional and biological markers of neuroplasticity and potential changes in frailty status. This approach aims at identifying the underlying pathogenic mechanisms of frailty and the results may add to previous interventional studies by offering new possible explanations for training effects found in frailty status and peripheral physical outcomes, such as muscle strength and muscle mass. Moreover, our study will provide new insights into how to prevent the continuous decline and avoid adverse outcomes in the development of frailty. This is important because frailty is a condition, which evolves from a subclinical state into a state of decompensation and ultimately overt frailty in the presence of stressors [103, 104]. Depending on one's preceding frailty state, transitions between frailty states may occur frequently over time 
and may also be recurrent, suggesting that frailty is a dynamic process [41]. By including frail as well as pre-frail subjects, we will be able to relate the training effects to changes in frailty status across a broad range of severity levels of pathological aging. In this context, our study might contribute to identify useful strategies, such as the maintenance of sensorimotor stimulation and function, for slowing progression in an early state and preserving older people's physical functions, autonomy, and quality of life that future studies can be based on.

\section{Strengths and limitations}

The present study has several strengths. First, the study includes an innovative multimodal sensorimotor approach designed to specifically target mechanisms of pathological neuroplasticity in frailty, compared to an active control condition. Second, participants will be recruited from the general population instead of clinical institutions. This will increase generalizability of the results and approach. Third, the treatment procedures are conceptualized as home-based training, thereby presumably increasing compliance for daily care.

The study is also subject to some limitations. Researchers and participants will not be blinded to treatment condition. However, by randomly assigning subjects to the treatment conditions and by using an appropriate active control group, we aim to eliminate any effects of expectations or systematic biases from inducing performance differences. The study design does not include follow-up assessments going beyond posttraining measurements after completion of the training, which should be addressed in future studies.

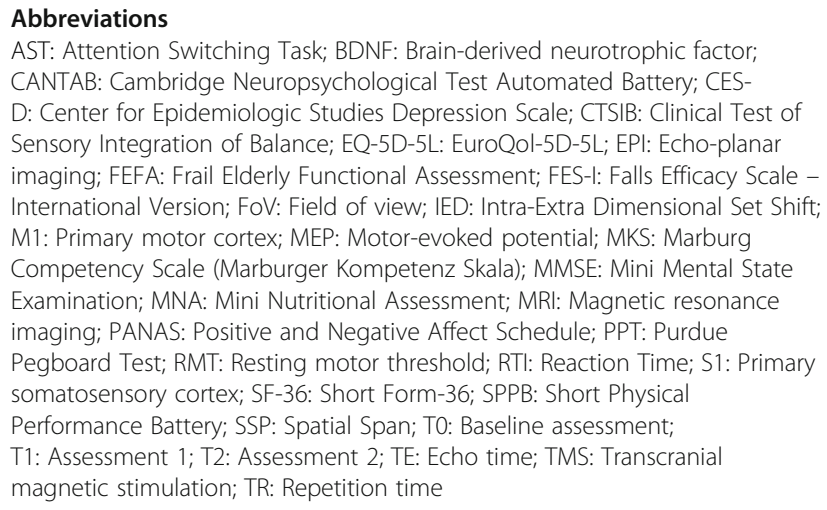

Abbreviations

AST: Attention Switching Task; BDNF: Brain-derived neurotrophic factor; CANTAB: Cambridge Neuropsychological Test Automated Battery; CESD: Center for Epidemiologic Studies Depression Scale; CTSIB: Clinical Test of Sensory Integration of Balance; EQ-5D-5L: EuroQol-5D-5L; EPI: Echo-planar imaging; FEFA: Frail Elderly Functional Assessment; FES-I: Falls Efficacy Scale International Version; FoV: Field of view; IED: Intra-Extra Dimensional Set Shift; M1: Primary motor cortex; MEP: Motor-evoked potential; MKS: Marburg Competency Scale (Marburger Kompetenz Skala); MMSE: Mini Mental State Examination; MNA: Mini Nutritional Assessment; MRI: Magnetic resonance imaging; PANAS: Positive and Negative Affect Schedule; PPT: Purdue Pegboard Test; RMT: Resting motor threshold; RTI: Reaction Time; S1: Primary somatosensory cortex; SF-36: Short Form-36; SPPB: Short Physical Performance Battery; SSP: Spatial Span; T0: Baseline assessment; T1: Assessment 1; T2: Assessment 2; TE: Echo time; TMS: Transcranial magnetic stimulation; TR: Repetition time

\section{Acknowledgements}

Not applicable.

\section{Authors' contributions}

HF conceived the study and HF and LF obtained funding. FB, ML, FN, LH, LF and $\mathrm{HF}$ contributed to the conception and design of the study. FB, ML and $\mathrm{LH}$ are involved in data acquisition. FB drafted the first version of the manuscript and ML, FN, LH, LF and HF edited and revised it and have approved its final version.

\section{Funding}

This work is supported by the Hector-Stiftung II. The funder had no role in study design, data collection and analysis, decision to publish, or preparation of the manuscript. Open Access funding enabled and organized by Projekt DEAL.

\section{Availability of data and materials}

The datasets produced by the completed study will be available from the corresponding author on reasonable request.

\section{Declarations}

Ethics approval and consent to participate

The study has been approved by the local Ethics Committee (Medical Ethics Committee II, Medical Faculty Mannheim, Heidelberg University; 2015-544 NMA) according to the Declaration of Helsinki. Participants are informed about all procedures and written informed consent is obtained prior to study inclusion.

\section{Consent for publication}

Not applicable.

\section{Competing interests}

The authors declare that they have no competing interests.

\section{Author details}

${ }^{1}$ Institute of Cognitive and Clinical Neuroscience, Central Institute of Mental Health, Medical Faculty Mannheim, Heidelberg University, Square J5, 68159 Mannheim, Germany. ${ }^{2}$ Institute of Medical Psychology and Medical Sociology, University Medical Center Schleswig-Holstein, Kiel University, Kiel, Germany. ${ }^{3}$ Department of Geriatric Psychiatry, Central Institute of Mental Health, Medical Faculty Mannheim, Heidelberg University, Mannheim, Germany. ${ }^{4}$ Department of Psychology, School of Social Sciences, University of Mannheim, Mannheim, Germany.

Received: 29 April 2021 Accepted: 20 May 2021

Published online: 03 June 2021

\section{References}

1. Clegg A, Young J, lliffe S, Rikkert MO, Rockwood K. Frailty in elderly people. Lancet. 2013;381(9868):752-62. https://doi.org/10.1016/S0140-6736(12)621 67-9.

2. Morley JE, Vellas B, Abellan van Kan G, Anker SD, Bauer JM, Bernabei R, et al. Frailty consensus: a call to action. J Am Med Dir Assoc. 2013;14(6):392-7. https://doi.org/10.1016/j.jamda.2013.03.022.

3. Ferrucci L, Cavazzini C, Corsi A, Bartali B, Russo CR, Lauretani F, et al. Biomarkers of frailty in older persons. J Endocrinol Investig. 2002;25(10 Suppl):10-5.

4. Dinse HR. Cortical reorganization in the aging brain. Prog Brain Res. 2006; 157:57-80. https://doi.org/10.1016/S0079-6123(06)57005-0.

5. Mahncke HW, Bronstone A, Merzenich MM. Brain plasticity and functional losses in the aged: scientific bases for a novel intervention. Prog Brain Res. 2006;157:81-109. https://doi.org/10.1016/S0079-6123(06)57006-2.

6. Rosano C, Studenski SA, Aizenstein HJ, Boudreau RM, Longstreth WT, Newman $A B$. Slower gait, slower information processing and smaller prefrontal area in older adults. Age Ageing. 2012;41(1):58-64. https://doi. org/10.1093/ageing/afr113.

7. Callisaya ML, Beare R, Phan TG, Chen J, Srikanth VK. Global and regional associations of smaller cerebral gray and white matter volumes with gait in older people. PLoS One. 2014;9(1):e84909. https://doi.org/10.1371/journal. pone.0084909.

8. Rodrigue KM, Raz N. Shrinkage of the entorhinal cortex over five years predicts memory performance in healthy adults. J Neurosci. 2004;24(4):95663. https://doi.org/10.1523/JNEUROSCI.4166-03.2004.

9. Head D, Rodrigue KM, Kennedy KM, Raz N. Neuroanatomical and cognitive mediators of age-related differences in episodic memory. Neuropsychology. 2008;22(4):491-507. https://doi.org/10.1037/0894-4105.22.4.491.

10. Sullivan EV, Rohlfing T, Pfefferbaum A. Quantitative fiber tracking of lateral and interhemispheric white matter systems in normal aging: relations to timed performance. Neurobiol Aging. 2010;31(3):464-81. https://doi.org/10.1 016/j.neurobiolaging.2008.04.007. 
11. Davis SW, Dennis NA, Buchler NG, White LE, Madden DJ, Cabeza R. Assessing the effects of age on long white matter tracts using diffusion tensor tractography. Neuroimage. 2009;46(2):530-41. https://doi.org/10.101 6/j.neuroimage.2009.01.068

12. Park DC, Polk TA, Park R, Minear M, Savage A, Smith MR. Aging reduces neural specialization in ventral visual cortex. Proc Natl Acad Sci U S A. 2004; 101(35):13091-5. https://doi.org/10.1073/pnas.0405148101.

13. Du Y, Buchsbaum BR, Grady CL, Alain C. Increased activity in frontal motor cortex compensates impaired speech perception in older adults. Nat Commun. 2016;7:1-12.

14. Carp J, Park J, Hebrank A, Park DC, Polk TA. Age-related neural dedifferentiation in the motor system. PLoS One. 2011;6(12):e29411. https:// doi.org/10.1371/journal.pone.0029411.

15. Koen JD, Hauck N, Rugg MD. The relationship between age, neural differentiation, and memory performance. J Neurosci. 2019;39(1):149-62. https://doi.org/10.1523/JNEUROSCI.1498-18.2018.

16. Goh JO. Functional dedifferentiation and altered connectivity in older adults: neural accounts of cognitive aging. Aging Dis. 2011;2(1):30-48.

17. Seidler RD, Bernard JA, Burutolu TB, Fling BW, Gordon MT, Gwin JT, et al. Motor control and aging: links to age-related brain structural, functional, and biochemical effects. Neurosci Biobehav Rev. 2010;34(5):721-33. https:// doi.org/10.1016/j.neubiorev.2009.10.005.

18. Sleimen-Malkoun R, Temprado JJ, Hong SL. Aging induced loss of complexity and dedifferentiation: consequences for coordination dynamics within and between brain, muscular and behavioral levels. Front Aging Neurosci. 2014;6:140140

19. Melendez Rl, Gregory ML, Bardo MT, Kalivas PW. Impoverished rearing environment alters metabotropic glutamate receptor expression and function in the prefrontal cortex. Neuropsychopharmacology. 2004;29(11): 1980-7. https://doi.org/10.1038/sj.npp.1300507.

20. Beaulieu C, Colonnier M. Number and size of neurons and synapses in the motor cortex of cats raised in different environmental complexities. J Comp Neurol. 1989;289(1):178-87. https://doi.org/10.1002/cne.902890115.

21. Rolland Y, Abellan Van Kan G, Benetos A, Blain H, Bonnefoy M, Chassagne P, et al. Frailty, osteoporosis and hip fracture: causes, consequences and therapeutic perspectives. J Nutr Health Aging. 2008;12(5):335-46.

22. Flor H, Elbert T, Knecht S, Wienbruch C, Pantev C, Birbaumer N, et al. Phantom-limb pain as a perceptual correlate of cortical reorganization following arm amputation. Nature. 1995;375(6531):482-4. https://doi.org/1 $0.1038 / 375482 \mathrm{a} 0$.

23. Jabbour $G$, lancu HD, Mauriège $P$, Joanisse DR, Martin $L$. High-intensity interval training improves performance in young and older individuals by increasing mechanical efficiency. Phys Rep. 2017;5(7):e13232. https://doi. org/10.14814/phy2.13232.

24. Lazarus NR, Izquierdo M, Higginson IJ, Harridge SDR. Exercise deficiency diseases of ageing: the primacy of exercise and muscle strengthening as first-line therapeutic agents to combat frailty. JJ Am Med Dir Assoc. 2018; 19(9):741-3. https://doi.org/10.1016/j.jamda.2018.04.014

25. Haider S, Grabovac I, Dorner TE. Effects of physical activity interventions in frail and prefrail community-dwelling people on frailty status, muscle strength, physical performance and muscle mass - a narrative review. Wien Klin Wochenschr. 2019;131(11-12):244-54. https://doi.org/10.1007/s00508-01 9-1484-7.

26. Cotman CW, Berchtold NC. Exercise: a behavioral intervention to enhance brain health and plasticity. Trends Neurosci. 2002;25(6):295-301. https://doi. org/10.1016/S0166-2236(02)02143-4.

27. Lu B, Nagappan G, Guan X, Nathan PJ, Wren P. BDNF-based synaptic repair as a disease-modifying strategy for neurodegenerative diseases. Nat Rev Neurosci. 2013;14(6):401401-16

28. Coelho FM, Pereira DS, Lustosa LP, Silva JP, Dias JMD, Dias RCD, et al, Physical therapy intervention (PTI) increases plasma brain-derived neurotrophic factor (BDNF) levels in non-frail and pre-frail elderly women. Arch Gerontol Geriatr. 2012;54(3):415-20. https://doi.org/10.1016/j.archger.2 011.05.014.

29. Puts MTE, Toubasi S, Andrew MK, Ashe MC, Ploeg J, Atkinson E, et al. Interventions to prevent or reduce the level of frailty in community-dwelling older adults: a scoping review of the literature and international policies. Age Ageing. 2017:46(3):383-92. https:/doi.org/10.1093/ageing/afw247.

30. Theou O, Stathokostas L, Roland KP, Jakobi JM, Patterson C, Vandervoort AA, et al. The effectiveness of exercise interventions for the management of frailty: a systematic review. J Aging Res. 2011;2011:569194.
31. de Labra C, Guimaraes-Pinheiro C, Maseda A, Lorenzo T, Millán-Calenti JC. Effects of physical exercise interventions in frail older adults: a systematic review of randomized controlled trials. BMC Geriatr. 2015;15(1):154. https:// doi.org/10.1186/s12877-015-0155-4.

32. Mahncke HW, Connor BB, Appelman J, Ahsanuddin ON, Hardy JL, Wood RA, et al. Memory enhancement in healthy older adults using a brain plasticitybased training program: a randomized, controlled study. Proc Natl Acad Sci U S A. 2006;103(33):12523-8. https://doi.org/10.1073/pnas.0605194103.

33. Shah T, Verdile G, Sohrabi H, Campbell A, Putland E, Cheetham C, et al. A combination of physical activity and computerized brain training improves verbal memory and increases cerebral glucose metabolism in the elderly. Transl Psychiatry. 2014;4(12):e487. https://doi.org/10.1038/tp.2014.122.

34. Anguera JA, Boccanfuso J, Rintoul JL, Al-Hashimi O, Faraji F, Janowich J, et al. Video game training enhances cognitive control in older adults. Nature. 2013:501(7465):97-101. https://doi.org/10.1038/nature12486.

35. Scheibe $S$, Carstensen LL. Emotional aging: recent findings and future trends. J Gerontol B Psychol Sci Soc Sci. 2010;65B(2):135-44. https://doi. org/10.1093/geronb/gbp132.

36. Coyle H, Traynor V, Solowij N. Computerized and virtual reality cognitive training for individuals at high risk of cognitive decline: systematic review of the literature. Am J Geriatr Psychiatry. 2015;23(4):335-59. https://doi.org/10.1 016/j.jagp.2014.04.009.

37. Bekrater-Bodmann R, Löffler A, Silvoni S, Frölich L, Hausner L, Desch S, et al. Tablet-based sensorimotor home-training system for amnestic mild cognitive impairments in the elderly: design of a randomised clinical trial. BMJ Open. 2019;9(8):e028632. https://doi.org/10.1136/bmjopen-2018-02 8632.

38. Nascimento CM, Pereira JR, Pires de Andrade L, Garuffi M, Ayan C, Kerr DS, et al. Physical exercise improves peripheral BDNF levels and cognitive functions in mild cognitive impairment elderly with different bdnf Val66Met genotypes. J Alzheimers Dis. 2015;43(1):81-91. https://doi.org/10.3233/JA D-140576.

39. Fried LP, Tangen CM, Walston J, Newman AB, Hirsch C, Gottdiener J, et al. Frailty in older adults: evidence for a phenotype. J Gerontol Med Sci. 2001; 56(3):146-56.

40. Chen X, Mao G, Leng SX. Frailty syndrome: an overview. Clin Interv Aging. 2014;9:433-41. https://doi.org/10.2147/CIA.S45300.

41. Gill TM, Gahbauer EA, Allore HG, Han L. Transitions between frailty states among community-living older persons. Arch Intern Med. 2006;166(4):41823. https://doi.org/10.1001/archinte.166.4.418.

42. Borkovec TD, Nau SD. Credibility of analogue therapy rationales. J Behav Ther Exp Psychiatry. 1972;3(4):257-60. https://doi.org/10.1016/0005-7916(72 )90045-6.

43. Faul F, Erdfelder $E$, Lang AG, Buchner A. G* power 3: a flexible statistical power analysis program for the social, behavioral, and biomedical sciences. Behav Res Methods. 2007;39(2):175-91. https://doi.org/10.3758/BF03193146.

44. Paraskevopoulos E, Herholz SC. Multisensory integration and neuroplasticity in the human cerebral cortex. Transl Neurosci. 2013:4(3):337-48.

45. Wallace MT, Stevenson RA. The construct of the multisensory temporal binding window and its dysregulation in developmental disabilities. Neuropsychologia. 2014;64:105-23. https://doi.org/10.1016/j. neuropsychologia.2014.08.005.

46. Darainy $M$, Vahdat S, Ostry DJ. Perceptual learning in sensorimotor adaptation. J Neurophysiol. 2013;110(9):2152-62. https://doi.org/10.1152/jn 00439.2013.

47. Censor N, Sagi D, Cohen LG. Common mechanisms of human perceptual and motor learning. Nat Rev Neurosci. 2012;13(9):658-64. https://doi.org/1 $0.1038 / n r n 3315$

48. Shmuelof L, Yang J, Caffo B, Mazzoni P, Krakauer JW. The neural correlates of learned motor acuity. J Neurophysiol. 2014;112(4):971-80. https://doi. org/10.1152/jn.00897.2013.

49. Brehmer $Y$, Kalpouzos G, Wenger E, Lövdén M. Plasticity of brain and cognition in older adults. Psychol Res. 2014;78(6):790-802. https://doi.org/1 0.1007/s00426-014-0587-z

50. Krohne HW, Egloff B, Kohlmann C-W, Tausch A. Untersuchungen mit eine deutschen version der "positive and negative affect schedule" (PANAS). [studies with a German version of the "positive and negative affect schedule" (PANAS)]. Diagnostica. 1996;42(2):139-56.

51. Watson D, Clark LA, Tellegen A. Development and validation of brief measures of positive and negative affect: the PANAS scales. J Pers Soc Psychol. 1988;54(6):1063-70. https://doi.org/10.1037/0022-3514.54.6.1063. 
52. Mitnitski AB, Graham JE, Mogilner AJ, Rockwood K. Frailty, fitness and latelife mortality in relation to chonological and biological age. BMC Geriatr. 2002;2(1):1. https://doi.org/10.1186/1471-2318-2-1.

53. Searle SD, Mitnitski A, Gahbauer EA, Gill TM, Rockwood K. A standard procedure for creating a frailty index. BMC Geriatr. 2008;8:24.

54. Malmstrom TK, Miller DK, Morley JE. A comparison of four frailty models. J Am Geriatr Soc. 2014;62(4):721-6. https://doi.org/10.1111/jgs.12735.

55. Woo J, Leung J, Morley JE. Comparison of frailty indicators based on clinical phenotype and the multiple deficit approach in predicting mortality and physical limitation. J Am Geriatr Soc. 2012;60(8):1478-86. https://doi.org/1 0.1111/j.1532-5415.2012.04074.x.

56. Blodgett J, Theou O, Kirkland S, Andreou P, Rockwood K. Frailty in NHANES: comparing the frailty index and phenotype. Arch Gerontol Geriatr. 2015; 60(3):464-70. https://doi.org/10.1016/j.archger.2015.01.016.

57. de Vries NM, Staal JB, van Ravensberg CD, Hobbelen JSM, Olde Rikkert MGM. Nijhuis-van der Sanden MWG. Outcome instruments to measure frailty: a systematic review. Ageing Res Rev. 2011;10(1):104-14. https://doi. org/10.1016/j.arr.2010.09.001.

58. Cesari M, Gambassi G, Van Kan GA, Vellas B. The frailty phenotype and the frailty index: different instruments for different purposes. Age Ageing. 2014; 43(1):10-2. https://doi.org/10.1093/ageing/aft160.

59. Caproni S, Muti M, Principi M, Ottaviano P, Frondizi D, Capocchi G, et al. Complexity of motor sequences and cortical reorganization in Parkinson's disease: a functional MRI Study. PLoS One. 2013;8(6):e66834. https://doi. org/10.1371/journal.pone.0066834.

60. Bekrater-Bodmann R, Foell J, Diers M, Kamping S, Rance M, Kirsch P, et al. The importance of synchrony and temporal order of visual and tactile input for illusory limb ownership experiences - an FMRI study applying virtual reality. PLoS One. 2014;9(1):e87013. https://doi.org/10.1371/journal.pone. 0087013.

61. Kolasinski J, Makin TR, Jbabdi S, Clare S, Stagg CJ, Johansen-Berg H. Investigating the stability of fine-grain digit Somatotopy in individual human participants. J Neurosci. 2016;36(4):1113-27. https://doi.org/10.1523/ JNEUROSCI.1742-15.2016.

62. Oliviero A, Profice P, Tonali PA, Pilato F, Saturno E, Dileone M, et al. Effects of aging on motor cortex excitability. Neurosci Res. 2006;55(1):74-7. https:// doi.org/10.1016/j.neures.2006.02.002.

63. Bhandari A, Radhu N, Farzan F, Mulsant BH, Rajji TK, Daskalakis ZJ, et al. A meta-analysis of the effects of aging on motor cortex neurophysiology assessed by transcranial magnetic stimulation. Clin Neurophysiol. 2016; 127(8):2834-45. https://doi.org/10.1016/j.clinph.2016.05.363.

64. McGregor KM, Carpenter H, Kleim E, Sudhyadhom A, White KD, Butler AJ, et al. Motor map reliability and aging: a TMS/fMRI study. Exp Brain Res. 2012;219(1):97-106. https://doi.org/10.1007/s00221-012-3070-3.

65. Guralnik JM, Simonsick EM, Ferrucci L, Glynn RJ, Berkman LF, Blazer DG, et al. A short physical performance battery assessing lower extremity function: association with self-reported disability and prediction of mortality and nursing home admission. J Gerontol Med Sci. 1994;49(2):M85-94. https://doi.org/10.1093/geronj/49.2.M85.

66. Kiely DK, Cupples LA, Lipsitz LA. Validation and comparison of two frailty indexes: the MOBILIZE Boston study. J Am Geriatr Soc. 2009;57(9):1532-9. https://doi.org/10.1111/j.1532-5415.2009.02394.x.

67. Study Investigators LIFE, Pahor M, Blair SN, Espeland M, Fielding R, Gill TM, et al. Effects of a physical activity intervention on measures of physical performance: results of the lifestyle interventions and independence for elders pilot (LIFE-P) study. J Gerontol A Biol Sci Med Sci. 2006;61(11):115765.

68. Shumway-Cook A, Horak F. Assessing the influence of sensory interaction of balance. Phys Ther. 1986;66(10):1548-50. https://doi.org/10.1093/ptj/66.10.1 548.

69. Tiffin J, Asher EJ. The Purdue pegboard; norms and studies of reliability and validity. J Appl Psychol. 1948;32(3):234-47. https://doi.org/10.1037/h0061266.

70. Desrosiers J, Hébert R, Bravo G, Dutil E. The purdue pegboard test: normative data for people aged 60 and over. Disabil Rehabil. 1995;17(5): 217-24. https://doi.org/10.3109/09638289509166638.

71. Brown M, Sinacore DR, Binder EF, Kohrt WM. Physical and performance measures for the identification of mild to moderate frailty. J Gerontol A Biol Sci Med Sci. 2000;55(6):M350-5. https://doi.org/10.1093/gerona/55.6.M350.

72. Bach M. The Freiburg visual acuity test - automatic measurement of visual acuity. Optom Vis Sci. 1996;73(1):49-53. https://doi.org/10.1097/00006324-1 99601000-00008.
73. Bach M. The Freiburg visual acuity test-variability unchanged by post-hoc re-analysis. Graefes Arch Clin Exp Ophthalmol. 2007;245(7):965-71. https:// doi.org/10.1007/s00417-006-0474-4

74. Bell-Krotoski JA, Fess EE, Figarola JH, Hiltz D. Threshold detection and Semmes-Weinstein monofilaments. J Hand Ther. 1995;8(2):155-62. https:// doi.org/10.1016/S0894-1130(12)80314-0.

75. Boyle PA, Buchman AS, Wilson RS, Leurgans SE, Bennett DA. Physical frailty is associated with incident mild cognitive impairment in community-based older persons. J Am Geriatr Soc. 2010;58(2):248-55. https://doi.org/10.1111/ j.1532-5415.2009.02671.x

76. Brigola AG, Rossetti ES, dos Santos BR, Neri AL, Zazzetta MS, Inouye K, et al. Relationship between cognition and frailty in elderly. Dement Neuropsychol. 2015;9(2):110-9. https://doi.org/10.1590/1980-57642015DN92 000005.

77. Gross AL, Xue QL, Bandeen-Roche K, Fried LP, Varadhan R, McAdamsDeMarco MA, et al. Declines and impairment in executive function predict onset of physical frailty. J Gerontol A Biol Sci Med Sci. 2016;71(12):1624-30. https://doi.org/10.1093/gerona/glw067.

78. O'Halloran AM, Finucane C, Savva GM, Robertson IH, Kenny RA. Sustained attention and frailty in the older adult population. J Gerontol B Psychol Sci Soc Sci. 2014;69(2):147-56. https://doi.org/10.1093/geronb/gbt009.

79. Gloth FM, Walston J, Meyer J, Pearson J. Reliability and validity of the frail elderly functional assessment questionnaire. Am J Phys Med Rehabil. 1995; 74(1):45-53. https://doi.org/10.1097/00002060-199501000-00008.

80. Gloth FM, Scheve AA, Shah S, Ashton R, McKinney R. The frail elderly functional assessment questionnaire: its responsiveness and validity in alternative settings. Arch Phys Med Rehabil. 1999;80(12):1572-6. https://doi. org/10.1016/S0003-9993(99)90332-5.

81. Hautzinger M, Bailer M, Hofmeister D, Keller F. Allgemeine Depressionsskala. 2nd ed. Göttingen: Hogrefe; 2012.

82. Radloff LS. The CES-D scale: a self report depression scale for research in the general population. Appl Psychol Meas. 1977;1(3):385-401. https://doi.org/1 $0.1177 / 014662167700100306$

83. Ware JE, Sherbourne CD. The MOS 36-Item short-form health survey (SF-36). Med Care. 1992;30(6):473-83. https://doi.org/10.1097/00005650-19920600000002.

84. Brooks R. EuroQol: the current state of play. Health Policy. 1996;37(1):53-72. https://doi.org/10.1016/0168-8510(96)00822-6.

85. Gauggel S, Peleska B, Bode RK. Relationship between cognitive impairments and rated activity restrictions stroke patients. J Head Trauma Rehabil. 2000; 15(1):710-23. https://doi.org/10.1097/00001199-200002000-00009.

86. Tinetti ME, Richman D, Powell L. Falls efficacy as a measure of fear of falling. J Gerontol. 1990;45(6):P239-43. https://doi.org/10.1093/geronj/45.6.P239.

87. McGee M, Jensen GL. Mini nutritional assessment (MNA): research and practice in the elderly. Am J Clin Nutr. 1999;71(1):158.

88. Rochat S, Cumming RG, Blyth F, Creasey H, Handelsman D, Le Couteur DG, et al. Frailty and use of health and community services by communitydwelling older men: the Concord health and ageing in men project. Age Ageing. 2010;39(2):228-33. https://doi.org/10.1093/ageing/afp257.

89. Sánchez-García S, Sánchez-Arenas R, García-Peña C, Rosas-Carrasco O, ÁvilaFunes JA, Ruiz-Arregui $L$, et al. Frailty among community-dwelling elderly Mexican people: prevalence and association with sociodemographic characteristics, health state and the use of health services. Geriatr Gerontol Int. 2014;14(2):395-402. https://doi.org/10.1111/ggi.12114.

90. Cesari M, Vellas B, Hsu FC, Newman AB, Doss H, King AC, et al. A physical activity intervention to treat the frailty syndrome in older persons - results from the LIFE-P study. J Gerontol A Biol Sci Med Sci. 2015;70(2):216-22. https://doi.org/10.1093/gerona/glu099.

91. Yamada M, Arai H, Sonoda T, Aoyama T. Community-based exercise program is cost-effective by preventing care and disability in Japanese frail older adults. J Am Med Dir Assoc. 2012;13(6):507-11. https://doi.org/10.101 6/j.jamda.2012.04.001.

92. Kim H, Suzuki T, Kim M, Kojima N, Ota N, Shimotoyodome A, et al. Effects of exercise and milk fat globule membrane (MFGM) supplementation on body composition, physical function, and hematological parameters in community-dwelling frail Japanese women: a randomized double blind, placebo-controlled, follow-up trial. PLoS One. 2015;10(2):e0116256. https:// doi.org/10.1371/journal.pone.0116256.

93. Kwon J, Yoshida Y, Yoshida H, Kim H, Suzuki T, Lee Y. Effects of a combined physical training and nutrition intervention on physical performance and health-related quality of life in prefrail older women living in the 
community: A randomized controlled trial. J Am Med Dir Assoc. 2015;16(3): 263.e1-263.e2638.

94. Ng TP, Feng L, Nyunt MSZ, Feng L, Niti M, Tan BY, et al. Nutritional, physical, cognitive, and combination interventions and frailty reversal among older adults: a randomized controlled trial. Am J Med. 2015;128(11):1225-36. https://doi.org/10.1016/.amjmed.2015.06.017.

95. Li CM, Chen CY, Li CY, Wang WD, Wu SC. The effectiveness of a comprehensive geriatric assessment intervention program for frailty in community-dwelling older people: a randomized, controlled trial. Arch Gerontol Geriatr. 2010;50(Suppl 1):S39-42. https://doi.org/10.1016/S0167-4 943(10)70011-X.

96. Mazya AL, Garvin P, Ekdahl AW. Outpatient comprehensive geriatric assessment: effects on frailty and mortality in old people with multimorbidity and high health care utilization. Aging Clin Exp Res. 2019; 31(4):519-25. https://doi.org/10.1007/s40520-018-1004-z.

97. Chen WT, Chou KH, Liu LK, Lee PL, Lee WJ, Chen LK, et al. Reduced cerebellar gray matter is a neural signature of physical frailty. Hum Brain Mapp. 2015;36(9):3666-76. https://doi.org/10.1002/hbm.22870.

98. Ryberg C, Rostrup E, Stegmann MB, Barkhof F, Scheltens P, van Straaten ECW, et al. Clinical significance of corpus callosum atrophy in a mixed elderly population. Neurobiol Aging. 2007;28(6):955-63. https://doi.org/10.1 016/j.neurobiolaging.2006.04.008.

99. Doba N, Tokuda Y, Goldstein NE, Kushiro T, Hinohara S. A pilot trial to predict frailty syndrome: the Japanese Health Research volunteer Study. Exp Gerontol. 2012;47(8):638-43. https://doi.org/10.1016/j.exger.2012.05.016.

100. Raji MA, Al Snih S, Ostir GV, Markides KS, Ottenbacher KJ. Cognitive status and future risk of frailty in older Mexican Americans. J Gerontol A Biol Sci Med Sci. 2010;65(11):1228-34.

101. Park H, Poo MM. Neurotrophin regulation of neural circuit development and function. Nat Rev Neurosci. 2013;14(1):7-23. https://doi.org/10.1038/ nrn3379.

102. Schäbitz WR, Steigleder T, Cooper-Kuhn CM, Schwab S, Sommer C, Schneider $\mathrm{A}$, et al. Intravenous brain-derived neurotrophic factor enhances poststroke sensorimotor recovery and stimulates neurogenesis. Stroke. 2007; 38(7):2165-72. https://doi.org/10.1161/STROKEAHA.106.477331.

103. Walston J, Hadley EC, Ferrucci L, Guralnik JM, Newman AB, Studenski SA, et al. Research agenda for frailty in older adults: toward a better understanding of physiology and etiology: summary from the American Geriatrics Society/National Institute on Aging research conference on frailty in older adults. J Am Geriatr Soc. 2006;54(6):991-1001. https://doi.org/1 0.1111/j.1532-5415.2006.00745.x.

104. Panza F, Lozupone M, Logroscino G. Understanding frailty to predict and prevent dementia. Lancet Neurol. 2019;18(2):133-4. https://doi.org/10.1016/ S1474-4422(18)30446-0.

\section{Publisher's Note}

Springer Nature remains neutral with regard to jurisdictional claims in published maps and institutional affiliations.

Ready to submit your research? Choose BMC and benefit from:

- fast, convenient online submission

- thorough peer review by experienced researchers in your field

- rapid publication on acceptance

- support for research data, including large and complex data types

- gold Open Access which fosters wider collaboration and increased citations

- maximum visibility for your research: over $100 \mathrm{M}$ website views per year

At BMC, research is always in progress.

Learn more biomedcentral.com/submissions 